\title{
Fluid Dynamics of Flapping Rigid and Spanwise-Flexible Elliptical Flat Plates at Low Reynolds Numbers
}

\author{
Jonathan M. Rausch ${ }^{1}$, Yeon Sik Baik ${ }^{2}$, Luis P. Bernal ${ }^{3} *$ Carlos E. S. Cesnik $^{4}$, Wei Shyy ${ }^{5}$, \\ and Michael V. $\mathrm{Ol}^{6} * *$ \\ *University of Michigan, Ann Arbor, MI, 48109 \\ **U.S. Air Force Research Laboratory, Wright-Patterson Air Force Base, OH 45433, U.S.A.
}

Presented here is an experimental investigation of sinusoidal pl unging rigid and flexible elliptical flat plates at Reynolds number $5.3 \times \mathbf{1 0}^{3}$. The sinusoidal plunging motion is characterized by the normalized plunge amplitude 0.175 , and the Strouhal number 0.203 . The investigation varies flexibility by varying the $\Pi_{1}$-parameter by varying the thickness of the models while maintaining the planform shape, the root chord $79 \mathrm{~mm}$, and the span 241 mm. A range of $\Pi_{1}$ was used from 30 to 90,240 . Dye flow visualization was performed using a 7-pronged rake oriented in the plane of the chord. Two camera vie ws are used for dye flow visualization, one is normal to the plane of the chord and the other is perpendicular to the plane's nor mal. Structural deformations were performed with Laser Doppler vibrometry (LDV). Deformations were sampled at the quarter and three-quarter chord location at $0 \%$, $25 \%, 50 \%$ and $75 \%$ span. Particle image velocimetry (PIV) measurements have been performed at $50 \%$ and $75 \%$ span at $30^{\circ}$ phase increments of the motion. Results show that a flexibility effect is only observed at $\Pi_{1}=30$. The flexibility effect is marked by bending $(40 \%$ of motion's root amplitude at $75 \%$ span $)$ and a phase lag $\left(60^{\circ}\right.$ phase at $75 \%$ span $)$.

\section{Nomenclature}

$\begin{array}{ll}A R & \text { aspect ratio } \\ b & \text { span [mm] } \\ c & \text { root chord [mm] } \\ D & \text { flat plate bending stiffness }=E t^{3} / 12\left(1-v^{2}\right),[\mathrm{N} \cdot \mathrm{m}] \\ E & \text { modulus of elasticity [GPa] } \\ f & \text { frequency [Hz] } \\ f^{*} & \text { modal frequency normalized by the motion frequency } \\ h & \text { plunge amplitude of oscillation [mm] } \\ h_{o} & \text { reduced plunge amplitude of oscillation } \\ k & \text { reduced frequency, } k=\pi f c / U_{\infty} \\ R e & \text { Reynolds number, } R e=c U_{\infty} / v \\ S t & \text { Strouhal number, } S t=2 f h_{o} / U_{\infty}=2 k h_{o} / \pi \\ t & \text { thickness [mm] } \\ t / c & \text { thickness-to-chord ratio }(\%) \\ U & \text { velocity [m/s] }\end{array}$

\footnotetext{
${ }^{1}$ Graduate Research Assistant, University of Michigan, Department of Aerospace Engineering, rauschjm@umich.edu

${ }^{2}$ Graduate Research Assistant, University of Michigan, Department of Aerospace Engineering, yeonb@umich.edu

${ }^{3}$ Associate Professor, University of Michigan, Department of Aerospace Engineering, lpb@umich.edu

${ }^{4}$ Professor, University of Michigan, Department of Aerospace En gineering, cesnik@ umich.edu

${ }^{5}$ Clarence L. "Kelly" Johnson Collegiate Professor and Chair, University of Michigan, Dep artment of Aerospace Engineering, weishyy@umich.edu

${ }^{6}$ Aerospace Engineer, Air Vehicles Directorate, Wright-Patterson AFB, Michael.O1@wpafb.af.mil
} 


$\begin{array}{ll}x & \text { Cartesian } x \text {-coordinate }[\mathrm{mm}] \\ \frac{x}{c} & \text { chord normalized } x \text {-coordinate } \\ y & \text { Cartesian } y \text {-coordinate }[\mathrm{mm}] \\ \frac{y}{c} & \text { chord normalized } y \text {-coordinate }\end{array}$

\section{Greek Sy mbols}

$\begin{array}{ll}\alpha_{e f f} & \text { effective angle of attack [deg.] } \\ \alpha_{0} & \text { mean angle of attack [deg.] } \\ v & \text { Poisson's ratio } \\ \Pi_{1} & \text { ratio of elastic and aerodynamic loading, } \Pi_{1}=D /\left(\rho_{\infty} U_{\infty}^{2} c^{3}\right) \\ \rho & \text { density, }\left[\mathrm{kg} / \mathrm{m}^{3}\right] \\ \phi & \text { positive phase offset for pitch relative to plunge [rad.] } \\ \omega & \text { vorticity [1/s] } \\ \omega^{*} & \text { normalized vorticity, } \omega^{*}=\omega c / U_{\infty}\end{array}$

$\underline{\text { Subscripts }}$

flexible, refers to $t / c=1 \%$

$r \quad$ rigid, refers to $t / c=4 \%$

$\infty$

freestream condition

\section{Introduction}

Recent interest in Micro Air Vehicles (MAVs) has created a desire to understand flapping wing flight because of natural flyers ${ }^{11}$ maneuverability and hovering ability. The bulk of flapping wing research to date has been focused on rigid wings. Experimental and computational investigations of rigid wings using pure plunge ${ }^{2,3}$, pure pitch ${ }^{4}$, and combined pitch/plunge ${ }^{5,6}$ kinematics have been performed in abundance. In contrast, wing flexibility and the resulting fluid-structure interaction have remained relatively unexplored in flapping wing research. Examining nature reveals the importance of flexibility to fish ${ }^{7}$ and stiffness variation present in insect wings ${ }^{8}$ prompts research into the flexibility's fluid dynamic significance.

The monograph by Shyy, et $\mathrm{al}^{9}$ provides a review of the low-Reynolds number aerodynamics and fluid-structure interaction for flapping wing flight. A review of the scaling parameters for the fluid-structure interaction of spanwise-flexible flapping wings is provided in Shyy, et $a l^{10}$. For this study the relevant scaling parameter is the ratio of elastic and aerodynamic forces given by Equation 1. This scaling parameter is the ratio of the equivalent flat plate stiffness to the aerodynamic loading.

$$
\Pi_{1}=\frac{D}{\rho_{\infty} U_{\infty}^{2} c^{3}}
$$

Recent experimental investigations into chordwise ${ }^{11}$ and spanwis $\mathrm{e}^{12}$ fle xibility have shown moderate increases in flexibility results in increased propulsive efficiency. Additionally, a recent combined experimental and computational study ${ }^{13}$ has shown how aerodynamic force generation can be enhanced via the introduction of wing fle xibility.

The focus of this study is the effect of spanwise fle xibility on the fluid dynamics of plunging isotropic wings. By fixing the kinematics and Reynolds number the effect of flexibility on an elliptical flat plate can be scientifically investigated. The details of the experimental investigation along with relevant scaling parameters will be provided in the following.

The sinusoidal plunging motion is characterized by a normalized plunge of 0.175 and a Strouhal number of 0.203. This Strouhal number lies within the range of Strouhal numbers $(0.2<S t<0.4)$ used by natural flapping wing flyers ${ }^{14}$. This Strouhal number range has been found to provide high propulsive efficiencies for spanwisefle xible wings undergoing a sinusoidal plunging motion ${ }^{12}$.

Plots of the normalized plunging amplitude and effective angle of attack as a function of the motion's phase for this study are provided in Figure 1. This induced effective angle of attack causes large excursions in effective angle of attack that go far beyond the static stall angle of attack for a flat plate as shown in Figure $1 \mathrm{~b}$. 


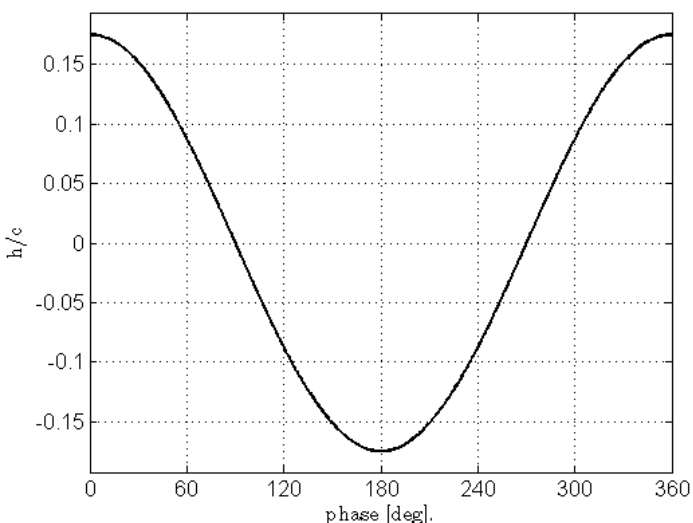

(a)

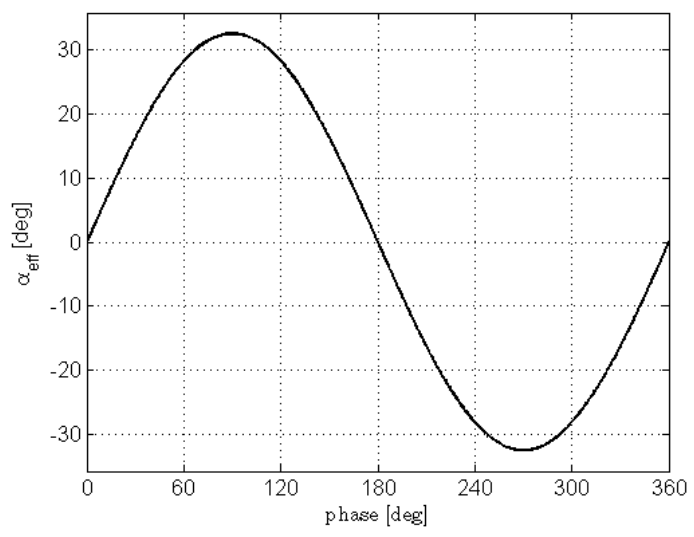

(b)

Figure 1. Plots of the (a) normalized plunge amplitude as function of motion phase and (b) effective angle of attack as a function of motion phase

Dye flow visualization, laser Doppler vibrometry (LDV), and particle image velocimetry (PIV) have been performed on four elliptical-planform flat-plate wing models with square edges. Two models are made of aluminum and two are made of high-density polyethylene (HDPE). There are two thicknesses used for each model material and the term rigid throughout this paper refers to a thickness $\mathrm{t} / \mathrm{c}$ of $4 \%$ and the term flexible throughout this paper refers to a thickness $\mathrm{t} / \mathrm{c}$ of $1 \%$.

Table 1 summarizes the kinematics, geometric properties, and material properties; and Table 2 summarizes the relevant kine matic scaling parameters, and

Table 3 summarizes the relevant fluid-structure interaction scaling parameters .

Table 1. Geometric, kinematic, and material properties

\begin{tabular}{|c|c|c|c|c|c|c|c|c|c|c|c|c|c|c|}
\hline \multicolumn{5}{|c|}{ geometric properties } & \multicolumn{4}{|c|}{ kinematic properties } & \multicolumn{3}{|c|}{$\begin{array}{c}\text { material properties - } \\
\text { aluminum }\end{array}$} & \multicolumn{3}{|c|}{$\begin{array}{c}\text { material properties - } \\
\text { HDPE }\end{array}$} \\
\hline $\begin{array}{c}b \\
{[\mathrm{~mm}]}\end{array}$ & $\begin{array}{c}c \\
{[\mathrm{~mm}]}\end{array}$ & $\begin{array}{c}t_{f} \\
{[\mathrm{~mm}]}\end{array}$ & $\begin{array}{c}\boldsymbol{t}_{r} \\
{[\mathrm{~mm}]}\end{array}$ & $A R$ & $\underset{[\mathbf{H z}]}{\boldsymbol{f}}$ & $\begin{array}{c}h \\
{[\mathrm{~mm}]}\end{array}$ & $\begin{array}{c}\boldsymbol{U}_{\infty} \\
{[\mathrm{mm} / \mathbf{s}]}\end{array}$ & $\begin{array}{c}\alpha_{o} \\
{[\mathrm{deg}]}\end{array}$ & $\begin{array}{c}E \\
{[\mathbf{G P a}]}\end{array}$ & $v$ & $\begin{array}{c}\rho \\
{\left[\mathbf{k g} / \mathbf{m}^{3}\right]}\end{array}$ & $\begin{array}{c}E \\
{[\mathbf{G P a}]}\end{array}$ & $v$ & $\begin{array}{c}\rho \\
{\left[\mathbf{k g} / \mathbf{m}^{3}\right]}\end{array}$ \\
\hline 241.3 & 79.4 & 0.794 & 3.175 & 7.65 & 0.489 & 13.78 & 67 & 0 & 70 & 0.3 & 2700 & 1.5 & 0.35 & 950 \\
\hline
\end{tabular}

Table 2. Dimensionless kinematic scaling parameters

\begin{tabular}{cccc}
$\boldsymbol{R e}$ & $\boldsymbol{k}$ & $\boldsymbol{S t}$ & $\boldsymbol{h}_{\boldsymbol{o}}$ \\
\hline 5,300 & 1.82 & 0.203 & 0.175 \\
\hline
\end{tabular}

3

American Institute of Aeronautics and Astronautics 
Table 3. Dimensionless fluid-structure scaling parameters

\begin{tabular}{ccccccc}
$(\boldsymbol{t} / \boldsymbol{c})_{\boldsymbol{f}}$ & $(\boldsymbol{t} / \boldsymbol{c})_{\boldsymbol{r}}$ & $\boldsymbol{A R}$ & $\begin{array}{c}\boldsymbol{\Pi}_{1, \boldsymbol{f}} \\
\boldsymbol{H D P E}\end{array}$ & $\begin{array}{c}\boldsymbol{\Pi}_{1, \boldsymbol{r}} \\
\mathbf{H D P E}\end{array}$ & $\begin{array}{c}\boldsymbol{\Pi}_{1, \boldsymbol{f}} \\
\boldsymbol{A L}\end{array}$ & $\begin{array}{c}\boldsymbol{\Pi}_{1, \boldsymbol{r}} \\
\boldsymbol{A L}\end{array}$ \\
\hline $1 \%$ & $4 \%$ & 7.65 & 30 & 1,960 & 1,460 & 90,240 \\
\hline
\end{tabular}

A structural modal analysis was performed to generate correlations between the modal frequencies and $\Pi_{1}$ parameter. The analysis was performed with SolidWorks using the geometric and material parameters of the four wings used in this study. The resulting first mode corresponded to the first bending mode and a least-squares regression was performed yielding the power fit in Equation 2.

$$
f^{*}=1.601\left(\Pi_{1}\right)^{0.391}
$$

The power fit in Equation 2 has a high correlation $\left(r^{2}=0.999\right)$. Equation 2 reveals that the normalized first modal frequency is never less than six for the $\Pi_{1}$-parameters tested. The modal analysis did not account for added mass effects.

\section{Flow Facility and Instrumentation}

The experimental facility is the University of Michigan's closed loop, free surface water channel. The water channel's test section is 24 " $\mathrm{x} 24$ " with glass sides and bottom. The water channel's freestream velocity capability ranges from $5 \mathrm{~cm} / \mathrm{s}$ to $40 \mathrm{~cm} / \mathrm{s}$. The water channel's turbulence intensity was measured using PIV and equals approximately $1 \%$.

The flat plate models are semi-spans with an endplate used as a symmetry plane. The model is actuated by a pitch-plunge oscillator rig above the water channel's free surface. The oscillator rig consists of a B4872TS Rotary Table (pitch simulation) and a Velmex 20-inch BiSlide (plunge simulation). The two motor stages are controlled by a Velmex VXM-1-1 motor controller.

\section{A. Dye Flow Visualization}

Dye flow visualization is a qualitative but insightful measure of a flow. The dye flow in the water channel is generated by a seven prong rake in the chordwise plane of the model at either the $50 \%$ or $75 \%$ span location. The dye flow streams are streak lines over the immersed flat plate. This experimental technique is useful for the streak lines are sensitive to boundary layer separation, leading and trailing edge vortices, and wake structure.

\section{B. Particle Image Velocimetry}

The PIV system is based on a double frame, single exposure design. A Quanta-Ray PIV Series Pulsed Nd:YAG laser creates a light sheet perpendicular to a charged-coupled device (CCD) array. The light sheet enters the test section side-window with the CCD array perpendicular to the light sheet and exposed to the chordwise cross section of the flat plate. Figure 2 illustrates the orientations of the PIV components. 


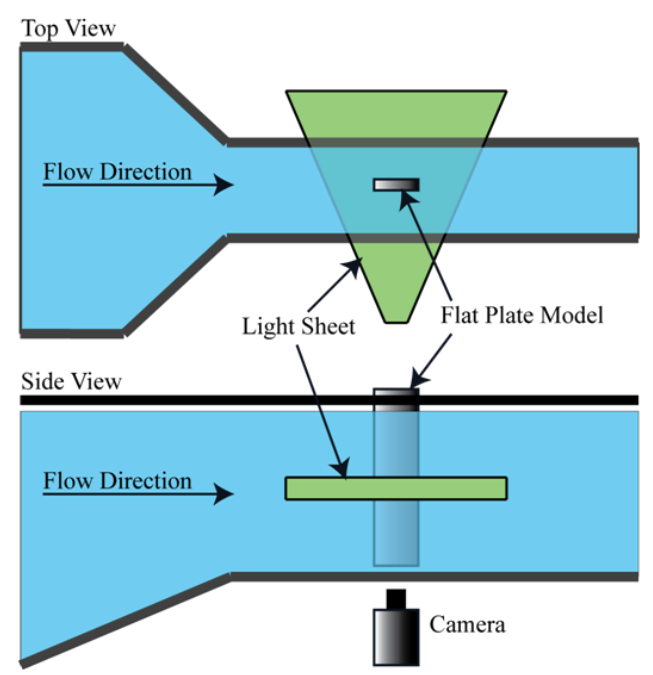

Figure 2. Diagram of the PIV setup with respect to the water channel's test section.

PIV was performed at the 50\% and $75 \%$ span which coincided with dye flow visualization planes. Figure 3 shows the coincident dye flow and PIV planes. In image in Figure 3 is from dye flow visualization with a superimposed green line at the $75 \%$ span location marking the coincident PIV plane.

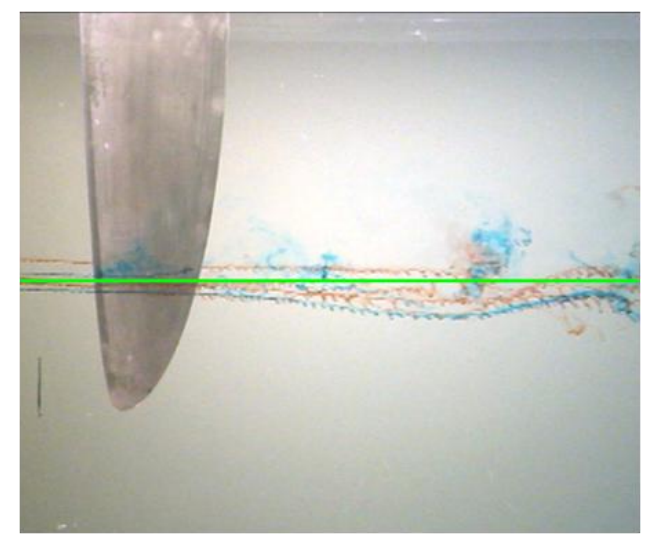

Figure 3. Coincident dye flow visualization and PIV planes (green line), flow is from left to right

A Cooke Corporation PCO. 4000 camera houses the $4008 \times 2672$ pixel CCD array used. The camera provides relatively high resolution for a given focal length lens.

The particles are Titanium Dioxide $\left(\mathrm{TiO}_{2}\right)$ with a particle diameter of $3 \mu \mathrm{m}$. Laser light intensity is adjusted to irradiate the particles providing the ideal particle image diameter, approximately $3 \times 3$ pixels for a $32 \times 32$ pixel interrogation window.

Table 4 provides a summary of the relevant parameters of the PIV measurements. 
Table 4. Table of PIV parameters

\begin{tabular}{|c|c|}
\hline \multicolumn{2}{|l|}{ Model } \\
\hline Chord, $\mathrm{c}[\mathrm{mm}]$ & 79 \\
\hline Span, b $[\mathrm{mm}]$ & 241 \\
\hline Flex. $t / c$ ratio $[\%]$ & 1 \\
\hline Rigid $t / c$ ratio $[\%]$ & 4 \\
\hline \multicolumn{2}{|l|}{ Flow } \\
\hline Flu id & Water \\
\hline $\operatorname{Re}$ & $5.3 \times 10^{3}$ \\
\hline$U_{\infty}[\mathrm{cm} / \mathrm{s}]$ & 6.7 \\
\hline \multicolumn{2}{|l|}{ Seeding } \\
\hline Type & $\mathrm{TiO}_{2}$ \\
\hline Partic le Physical Diameter $[\mu \mathrm{m}]$ & 3 \\
\hline Dispersant & DARVAN C-N \\
\hline \multicolumn{2}{|l|}{ Laser } \\
\hline Type & Nd:YAG \\
\hline Max Pulse Energy [mJ/pulse] & 200 \\
\hline \multicolumn{2}{|l|}{ Recording } \\
\hline FOV $\left[\mathrm{mm}^{2}\right]$ & $\cong 17,000$ \\
\hline Camera & PCO. 4000 \\
\hline Pixel Length Scale [pixels/mm] & 25 \\
\hline Dynamic Range & 16 bit \\
\hline f-nu mber & 5 \\
\hline Focal length $[\mathrm{mm}]$ & 105 \\
\hline Partic le Image Diameter [pixels] & 3 \\
\hline$\Delta t[\mathrm{~ms}]$ & 3.626 \\
\hline \multicolumn{2}{|l|}{ Image Pair Processing } \\
\hline High Resolution Window [pixels ${ }^{2}$ ] & $32 \times 32$ \\
\hline Low Resolution Window [pixels ${ }^{2}$ ] & $64 \times 64$ \\
\hline Sample Size* [min. - max.] & approx. $240-270$ \\
\hline Total PIV Realizations & 270 \\
\hline
\end{tabular}

* Sample size refers to the number of independent samples that remain after bad data points are replaced using a median filter; the sample size is given as a range from smallest to largest throughout the data.

\section{Laser Doppler Vibrometry}

For this flexible flapping wing experiment 'contact' measures (i.e. accelerometers) of the wing's deformation would not be appropriate; therefore Laser Doppler Vibrometry (LDV) was selected to quantify the structural deformation.

The Laser Doppler Vibrometer (LDV) measurements were performed with a Polytec PSV-400 Scanning Vibrometer ${ }^{15,16}$. The vibrometer system is composed of the PSV-I-400 laser head, the OFV-5000 controller, the PSV-E-401 junction box, and a desktop computer. The PSV-I-400 laser head uses a He-Ne laser to emit a $633 \mathrm{~nm}$ linear polarized beam. Using the principles of interferometry, the vibrometer system is able to yield velocities by measuring the interference pattern generated by the different path lengths between a reference beam and a backscattered object beam. The OFV-5000 is responsible for interpreting the interference pattern. The PSV-E-401 junction box is responsible for interfacing and synchronizing all hardware.

The vibrometer measured velocity at the quarter and three-quarter chord at the $0 \%, 25 \%, 50 \%$, and $75 \%$ span location. Figure 4 is an example of the $75 \%$ span location where Figure 4(a) is the three-quarter chord sampling location and Figure 4(b) is the quarter chord sampling location. In Figure 4 the freestream flows right-to-left. Additionally in Figure 4, reflective tape can be observed that is used to facilitate a diffuse reflection of the laser light. 


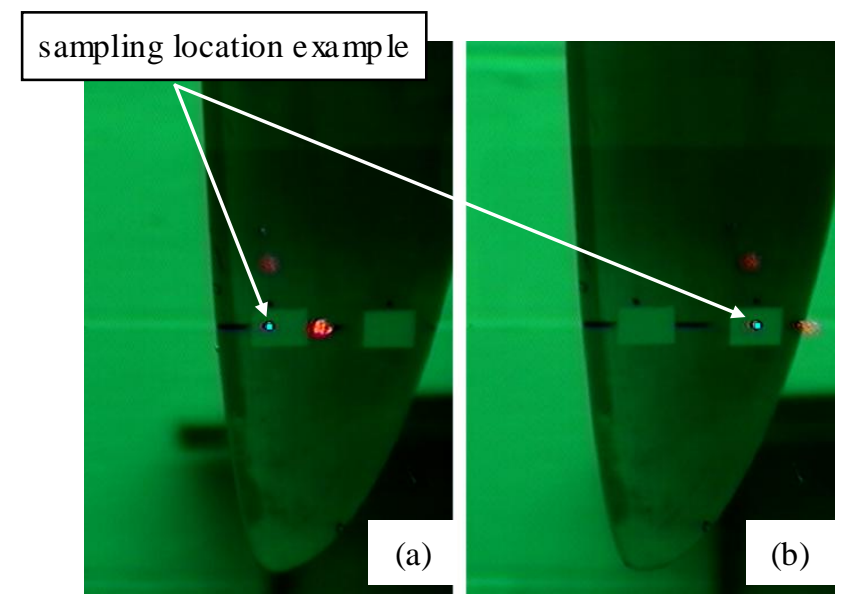

Figure 4. Laser vibrometer sampling locations, (a) is the quarter chord location at the $75 \%$ span location, (b) is the three-quarter chord location at the $75 \%$ span location

Since the backscattered laser light is passing through two mediums (water and air) a correction is necessary. A 0.75 scaling factor on the velocity measurement is used the correct for the mismatched indices of refraction.
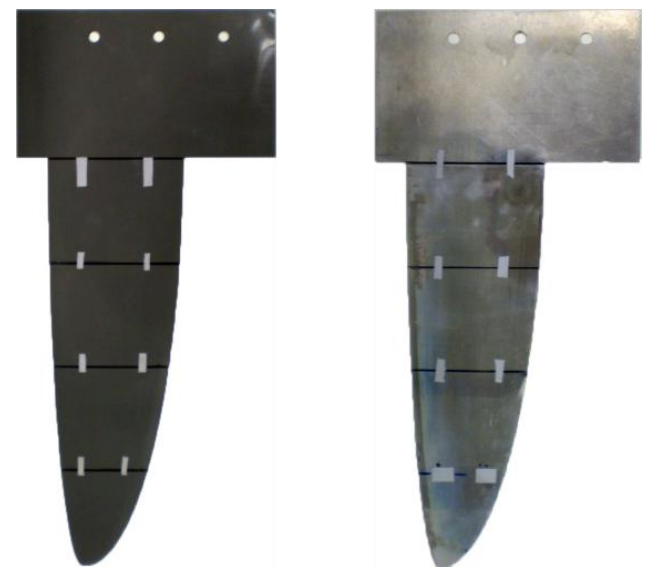

Figure 5. Aluminum (left) and HDPE (right) models with reflective tape at the eight sampling locations

Data was acquired over 25 periods of motion at $512 \mathrm{~Hz}$. The velocity data is interpolated and partitioned into 25 arrays. Each of the 25 interpolated arrays is numerically integrated to yield 25 displacement arrays. Both the velocity and displacement arrays are phase-averaged. The phase-averaged signals were smoothed with a multiple-pass symmetric moving average with three taps. The power spectra of the all the acquired signals were examined before smoothing to check that significant signals weren't excluded.

\section{Results and Discussion}

\section{A. Dye Flow Results}

Dye flow visualization results are provided in Figure 6. The most noticeable observation of the dye flow visualization is the significant deformation of the flexible HDPE model. A noticeable deformation is not observed for the other models.

All of the models show a leading edge vortex forming on top side of the wing during the downstroke. With the exception of the flexible HDPE model, the leading edge vortices for the other models have three-dimensional vortex cores that weakly move towards the root. The fle xible HDPE model, in contrast, has a three-dimensional vortex core that strongly moves towards the tip. 
Phase

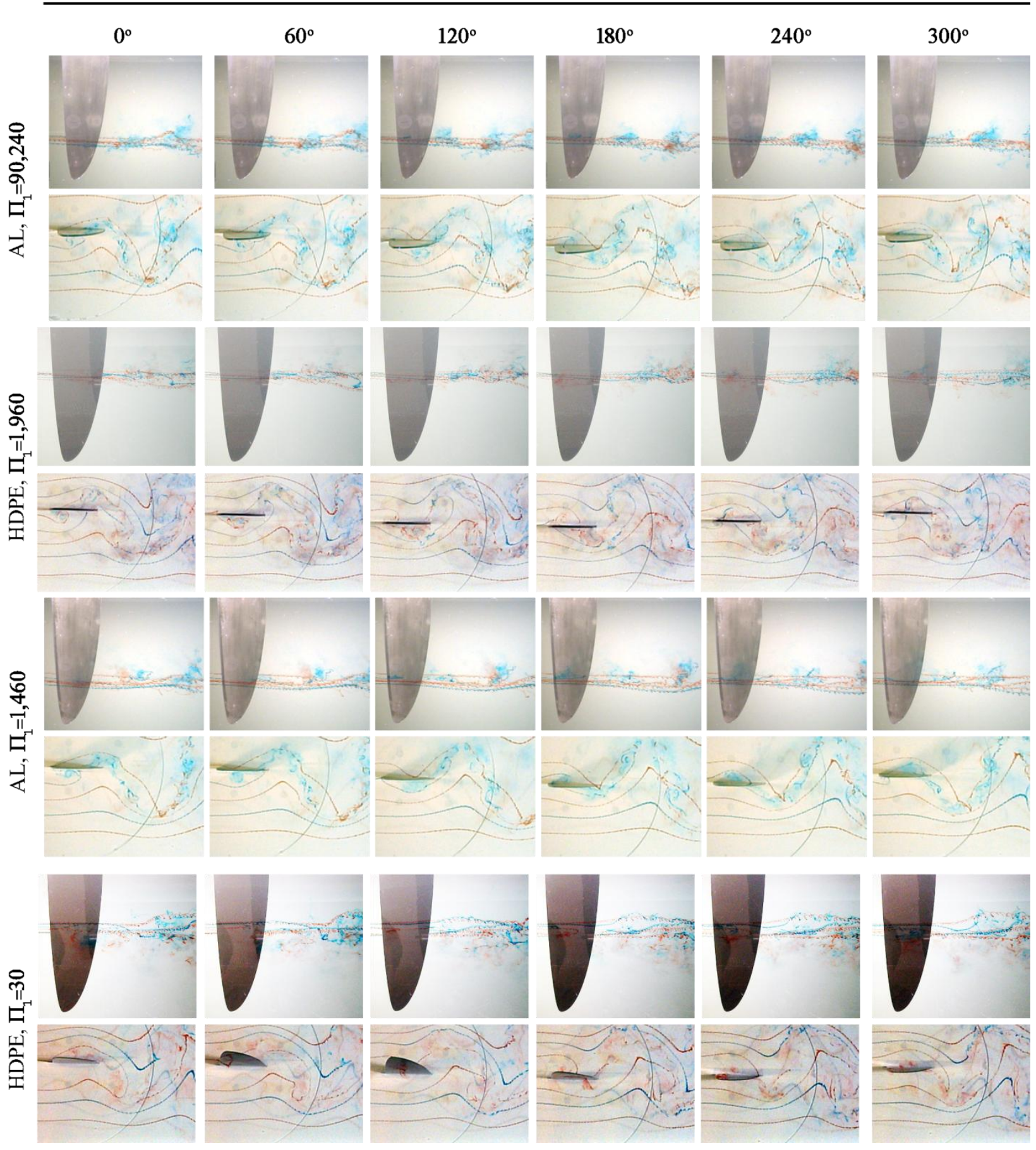

Figure 6. Dye flow visualization at $60^{\circ}$ phase increments comparing the flexible and rigid models. In these pictures the flow is from left to right.

\section{B. Vibrometer Results}

As previously stated the vibrometer measurements were at the quarter and three-quarter chord location at $0 \%$, $25 \%, 50 \%$, and $75 \%$ span. The data was acquired as velocity data and then numerically integrated to yield displacement. 
In the following data presentation the aluminum models are not included since they deformed the same or less than the rigid HDPE model.

The deformation measurements showed that only the model with the lowest $\Pi_{1}$-parameter significantly deformed. Figure 7 compares the deflection at each sampling location for the two HDPE models. Figure 7a shows the deformation of the lowest $\Pi_{1}$-para meter model to be $40 \%$ the amplitude of motion at the $75 \%$ span location. The model's deformation also lags the root motion by as much as $70^{\circ}$ at the $75 \%$ span location.

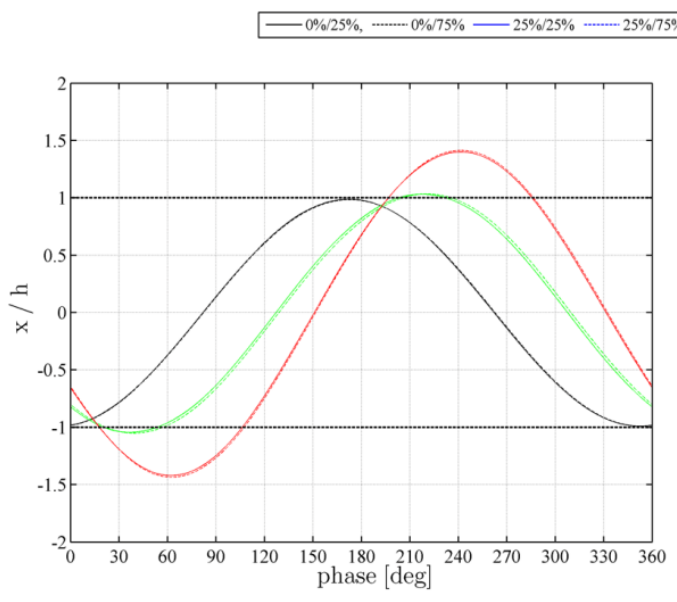

(a)

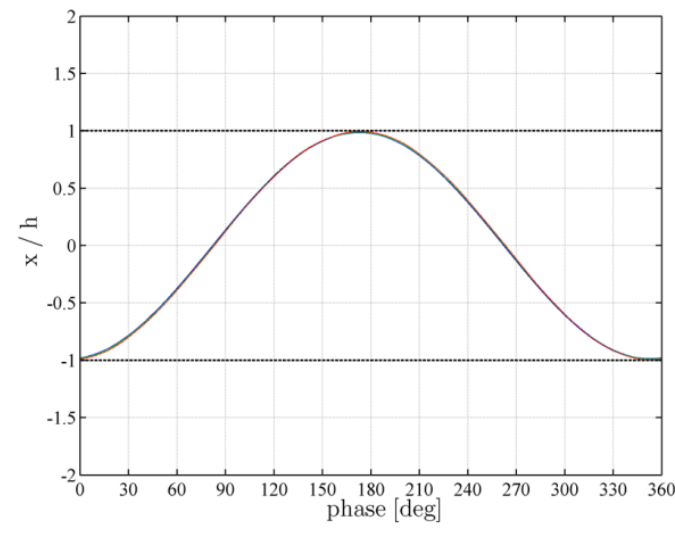

(b)

Figure 7. Deflection at each sampling location for the HDPE models (legend lists data as span\%/chord\%). (a) Flexible HDPE model $\left(\Pi_{1}=30\right)$, for this model the deformation lags the root by $70^{\circ}$. (b) Rigid HDPE model $\left(\Pi_{1}=1,960\right)$, for this model all of the sampling locations are in-phase and the deformations are insignificant.

The models were either solid aluminum or HDPE without additional reinforcement. This fact causes the elastic axis to follow the centroid of the model's cross-sectional area from root to tip. Given then prescribed planform shape the elastic axis is therefore slightly curved towards the leading edge. The curvature introduces a bend-twist coupling. In light of this effect and possible aerodynamic-twist the twist at each spanwise section was calculated from the deformation measurements. The twist calculation is plotted in Figure 8 where the lowest $\Pi_{1}$-parameter model has the most significant deformation. The twist in Figure $8 \mathrm{a}$ reaches a maximum of $0.75^{\circ}$ at $75 \%$ span and there is a $180^{\circ}$ phase shift between $50 \%$ and $75 \%$ span. In Figure $8 \mathrm{a}$ the RMS error was included because cycle-to-cycle variation was observed in the PIV images.

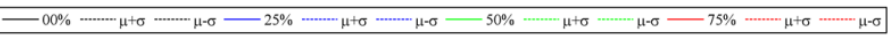

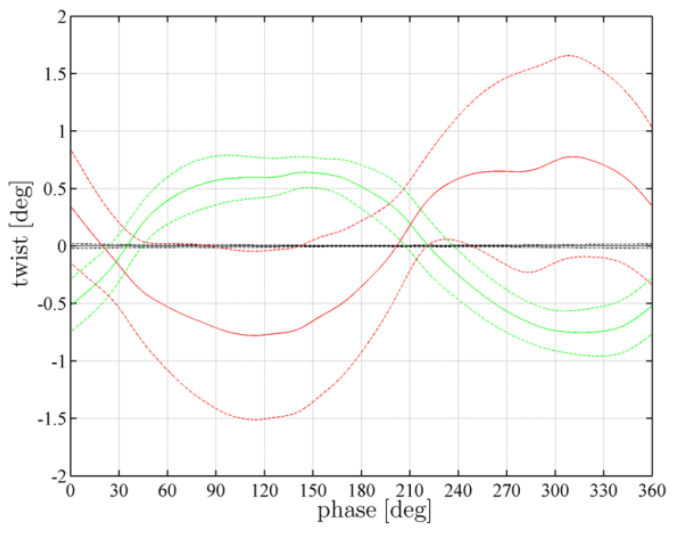

(a)

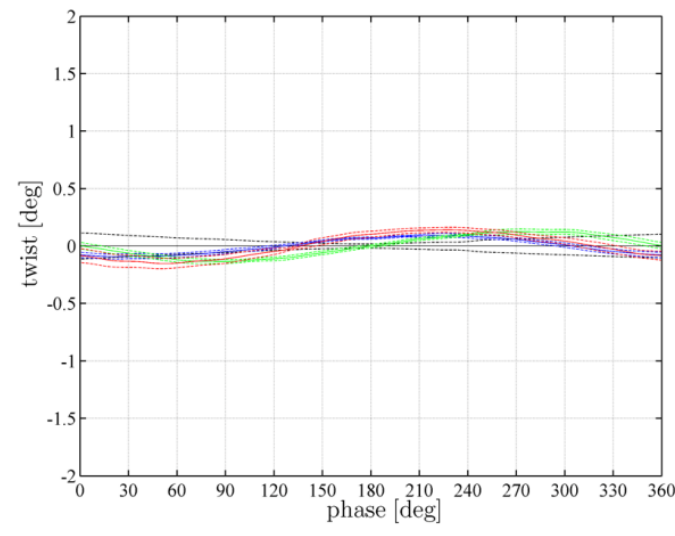

(b)

Figure 8. Mean sectional twist angle and RMS error at each phase . (a) Flexible HDPE model $\left(\Pi_{1}=30\right)$, mean twist angle reaches a maximum of 0.8 degrees with a large standard deviation. (b) Rigid HDPE model $\left(\Pi_{1}=1,960\right)$, mean twist angle is much smaller than flexible HDPE model.

9

American Institute of Aeronautics and Astronautics 
The geometric twist is dwarfed by the effective angle of attack generated by the plunge induced angle of attack. The peak effective angle of attack at the root is $32.5^{\circ}$. The spanwise deformation resulted in an even larger plunge induced angle of attack peak towards the tip (50\% span $-33.8^{\circ}, 75 \%$ span $\left.-41.7^{\circ}\right)$. Figure 9 plots the plunge induced angle of attack along the span highlighting the increase towards the tip.

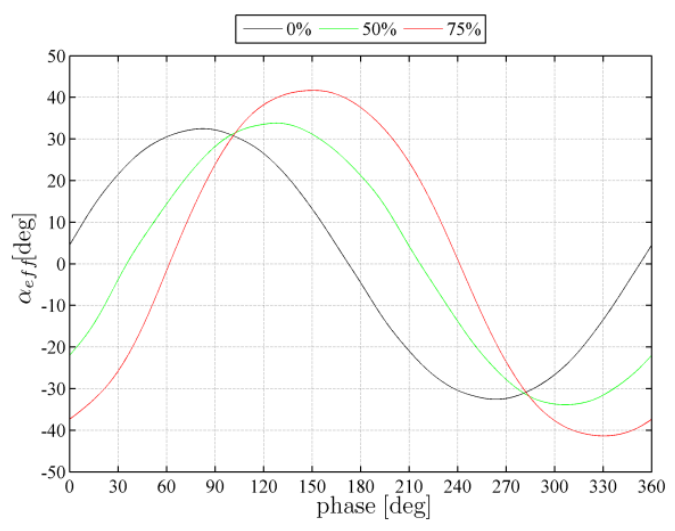

(a)

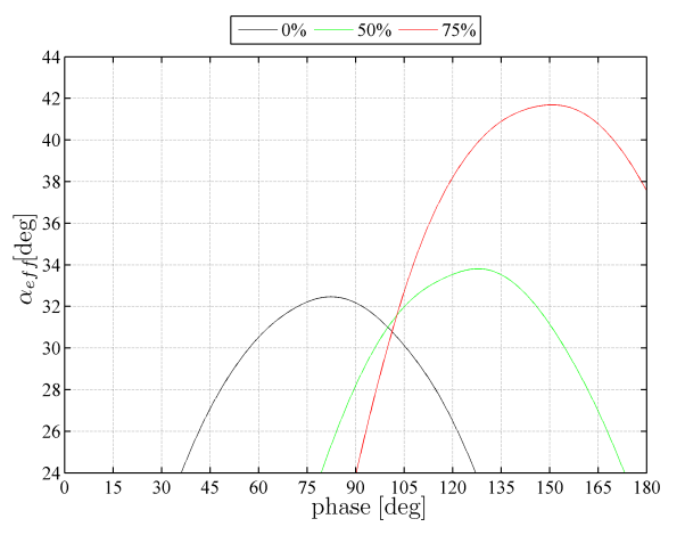

(b)

Figure 9. Plunge induced angle of attack at 0\%, 50\%, and 75\% span. (a) Axes show full sinusoidal oscillation of the plunge induced angle of attack. (b) Axes are refined to show peak effective angle of attack magnitude over half the period of motion.

\section{PIV Results}

The PIV results are presented as a comparison between the mean $x$-velocity and vorticity fields in Figure 10 through Figure 13 and a comparison between the mean vorticity and Reynolds stress fields in Figure 14 through Figure 17. The root chord was used as the reference length for normalization.

Concentrations of Reynolds stresses in Figure 14 through Figure 17 are isolated to shear layers, cycle-to-cycle variation in flow structure locations, and vortex interaction with the plate or shear-layers.

The flow topology of the rigid aluminum model is highlighted by a leading edge vortex that forms during the downstroke at the $50 \%$ and $75 \%$ span locations. The leading edge vortex then separates and advects towards the trailing edge. The lead ing edge vortex passes the trailing edge at an earlier phase for the $75 \%$ location.

The flow topology of the flexible alu minum model is highlighted by a leading edge vortex that forms during the downstroke at the $50 \%$ and $75 \%$ span locations. The leading edge vortex then separates and advects towards the trailing edge. The leading edge vortex for this model differs fro $m$ the rigid alu minum model since the vortex remains on the upper surface until a later phase of motion.

The flow topology of the rigid HDPE model is similar to the aluminum models since its $\Pi_{1}$ equals the same value as the flexible aluminum model. Therefore, the flow is dominated by the formation of a leading edge vortex during the downstroke that separates and advects towards the trailing edge. The path of the leading edge vortex towards the trailing edge is most similar to the rigid aluminum model with the leading edge vortex departing the 75\% span upper surface at approximately the same phase of motion. This similarity between flowfields indicates a dependence on the model thickness since no significant deformation was observed.

The flow topology of the flexible HDPE is the most unique since it underwent significant structural deformation. Similar to the other three models the flow is dominated by the formation of a leading edge vortex. For this model the deformations prevent the camera during PIV from observing the upper surface from $30^{\circ}$ to $180^{\circ}$ phase thus the early formation of the leading edge vortex is missing. As the leading edge vortex forms the $75 \%$ span location forms a significantly larger leading edge vortex. The larger leading edge vortex at the $75 \%$ span location is caused by the larger effective angle of attack as observed in Figure 9. 


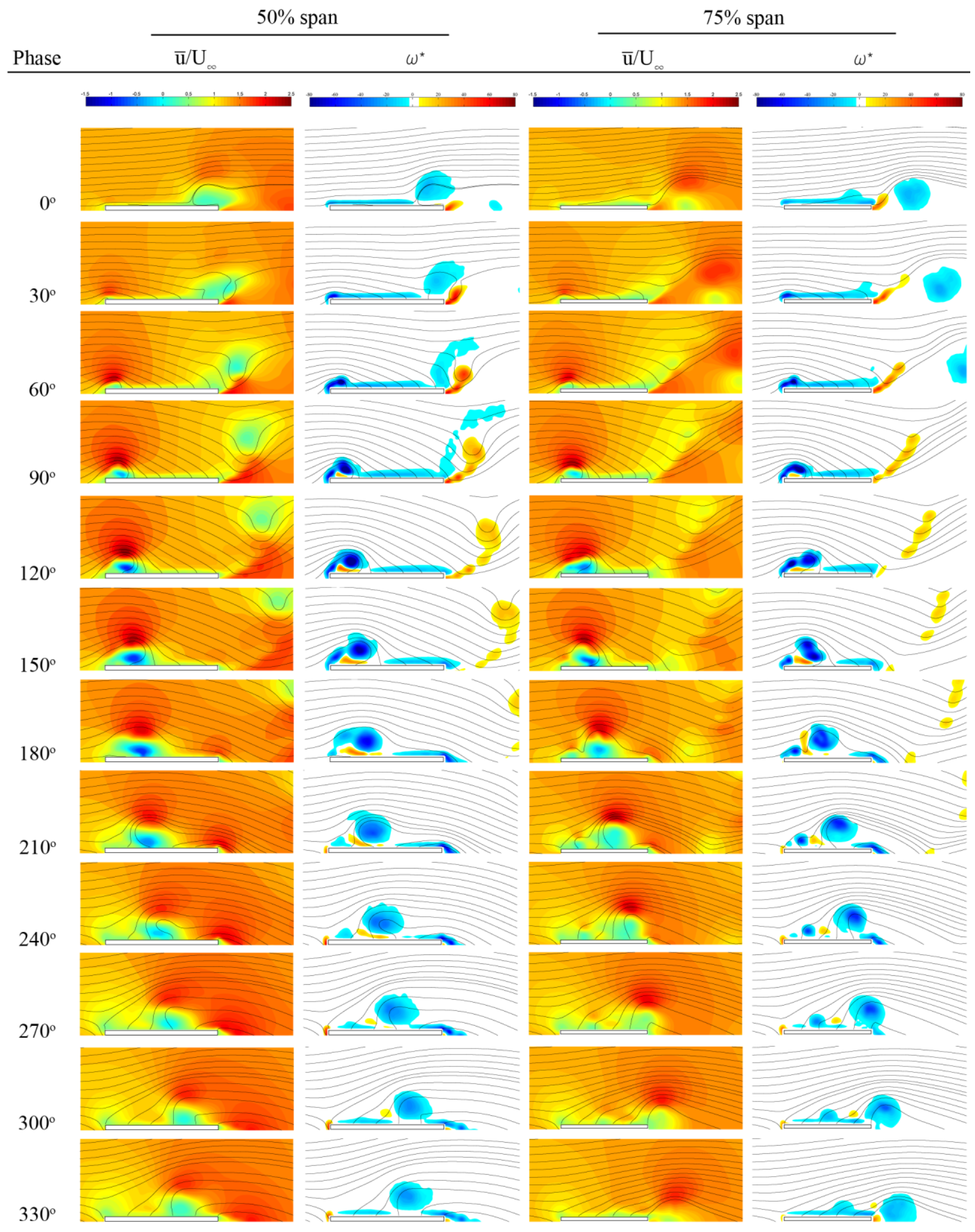

Figure 10. Normalized mean u-velocity and vorticity for rigid aluminum model $\left(\Pi_{1}=90,240\right)$. PIV was performed at $50 \%$ and $75 \%$ span. Flow is from the left to right. 


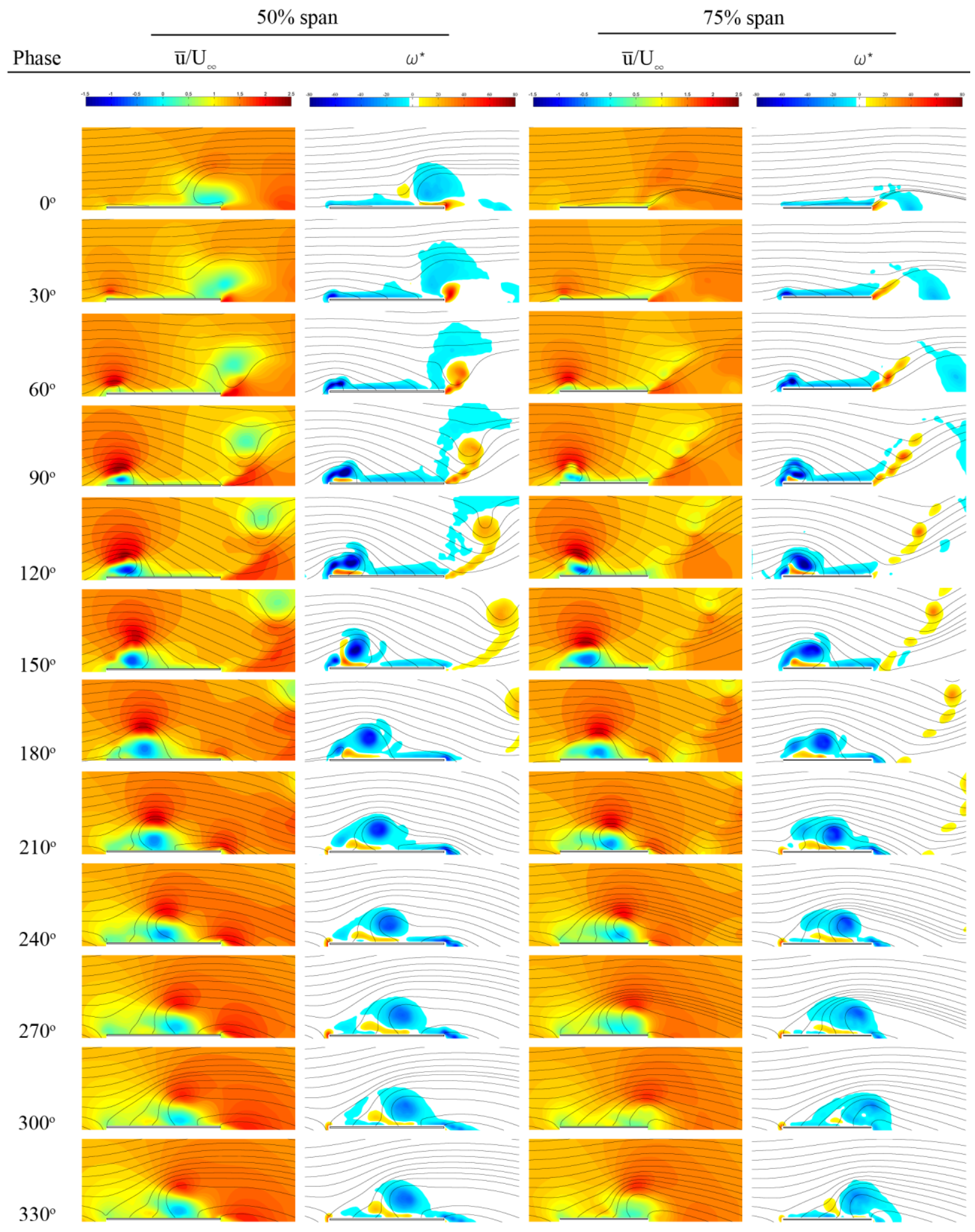

Figure 11. Normalized mean u-velocity and vorticity for flexible aluminum model $\left(\Pi_{1}=1,460\right)$. PIV was performed at $50 \%$ and $75 \%$ span. Flow is from the left to right.

12

American Institute of Aeronautics and Astronautics 


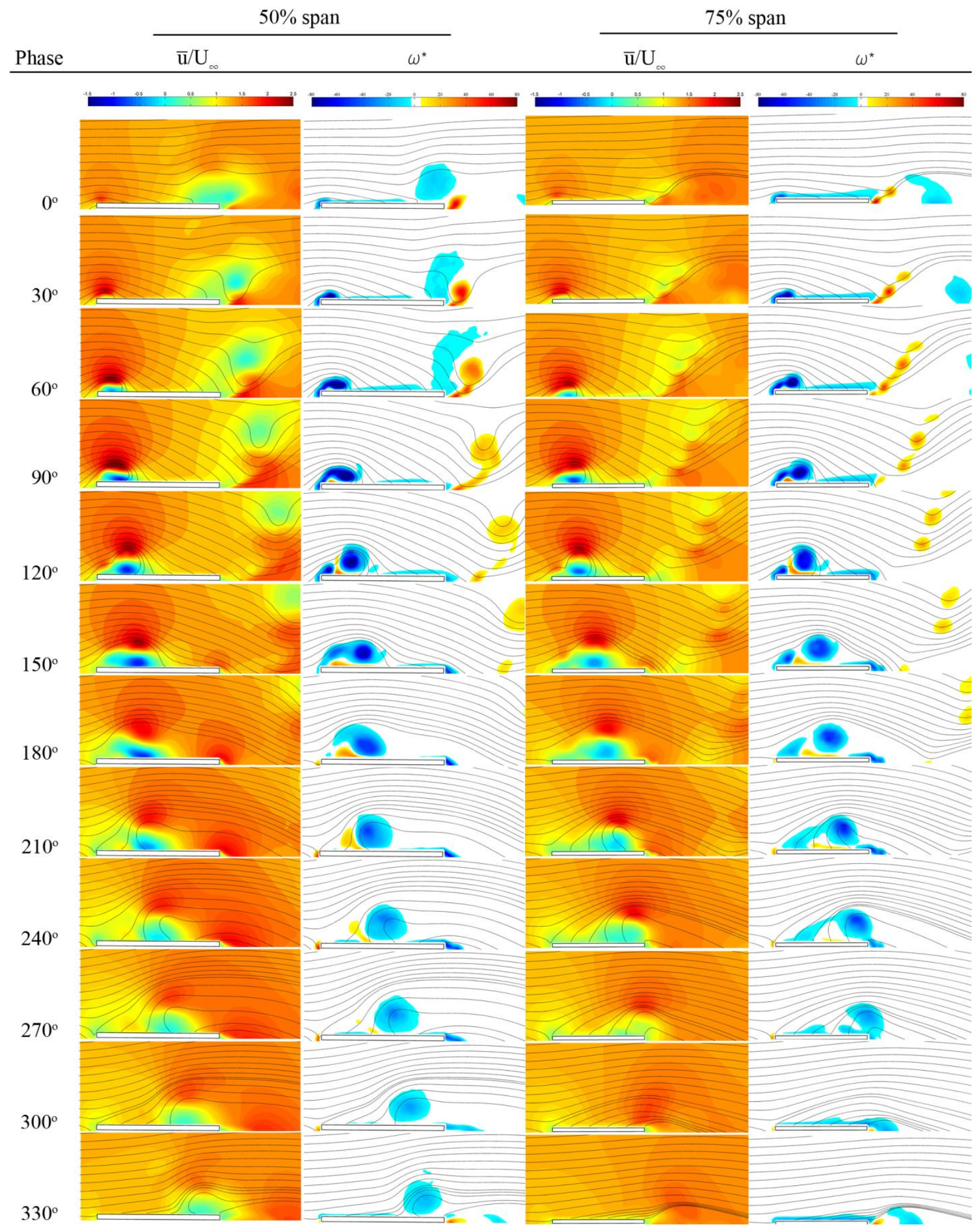

Figure 12. Normalized mean u-velocity and vorticity for rigid HDPE model $\left(\Pi_{1}=1,960\right)$. PIV was performed at 50\% and $75 \%$ span. Flow is from the left to right.

13

American Institute of Aeronautics and Astronautics 


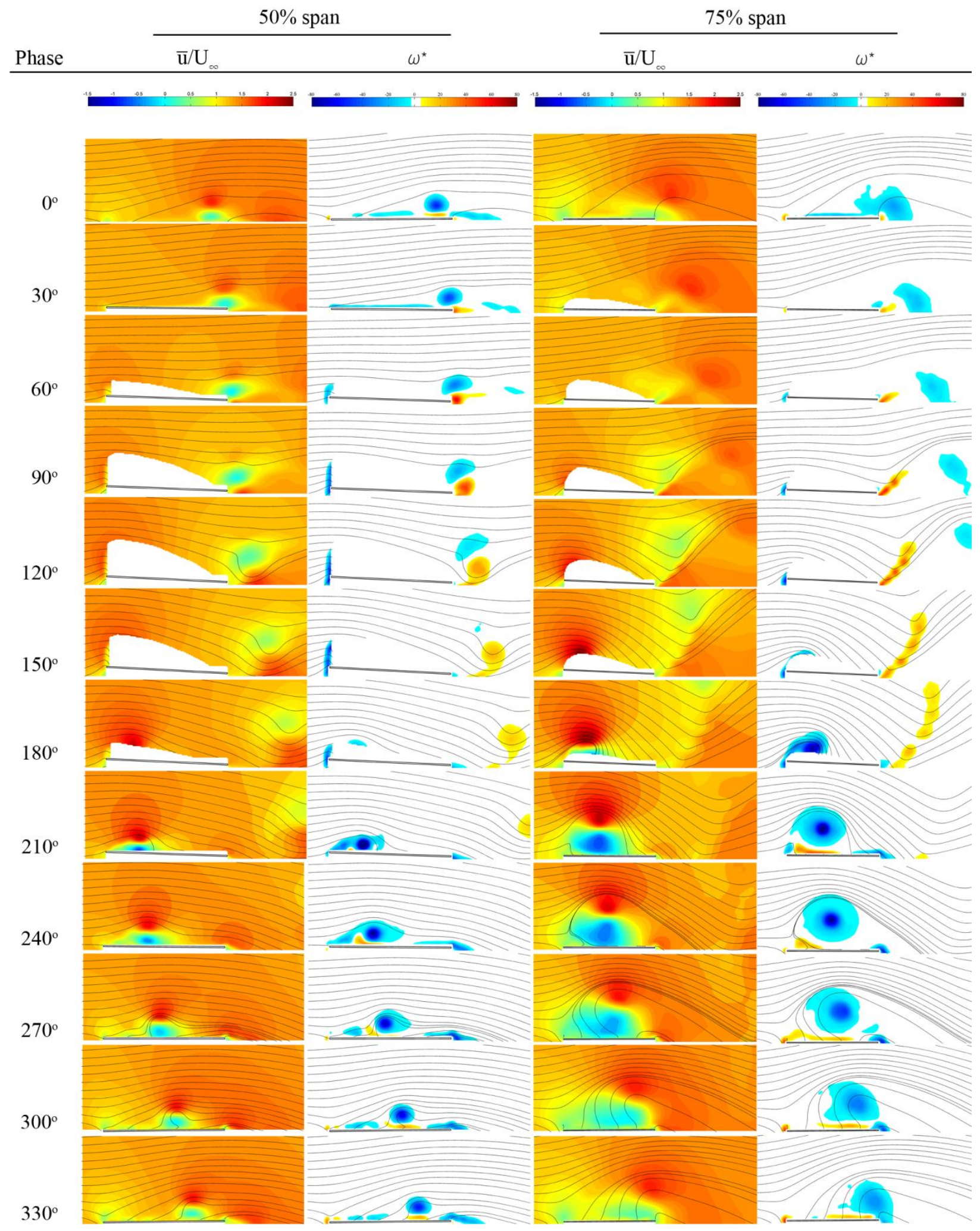

Figure 13. Normalized mean u-velocity and vorticity for flexible HDPE model $\left(\Pi_{1}=30\right)$. PIV was performed at $50 \%$ and $75 \%$ span. Portions of the flowfields for phase $30^{\circ}$ through $180^{\circ}$ are masked because of structural deformations. Flow is from the left to right. 


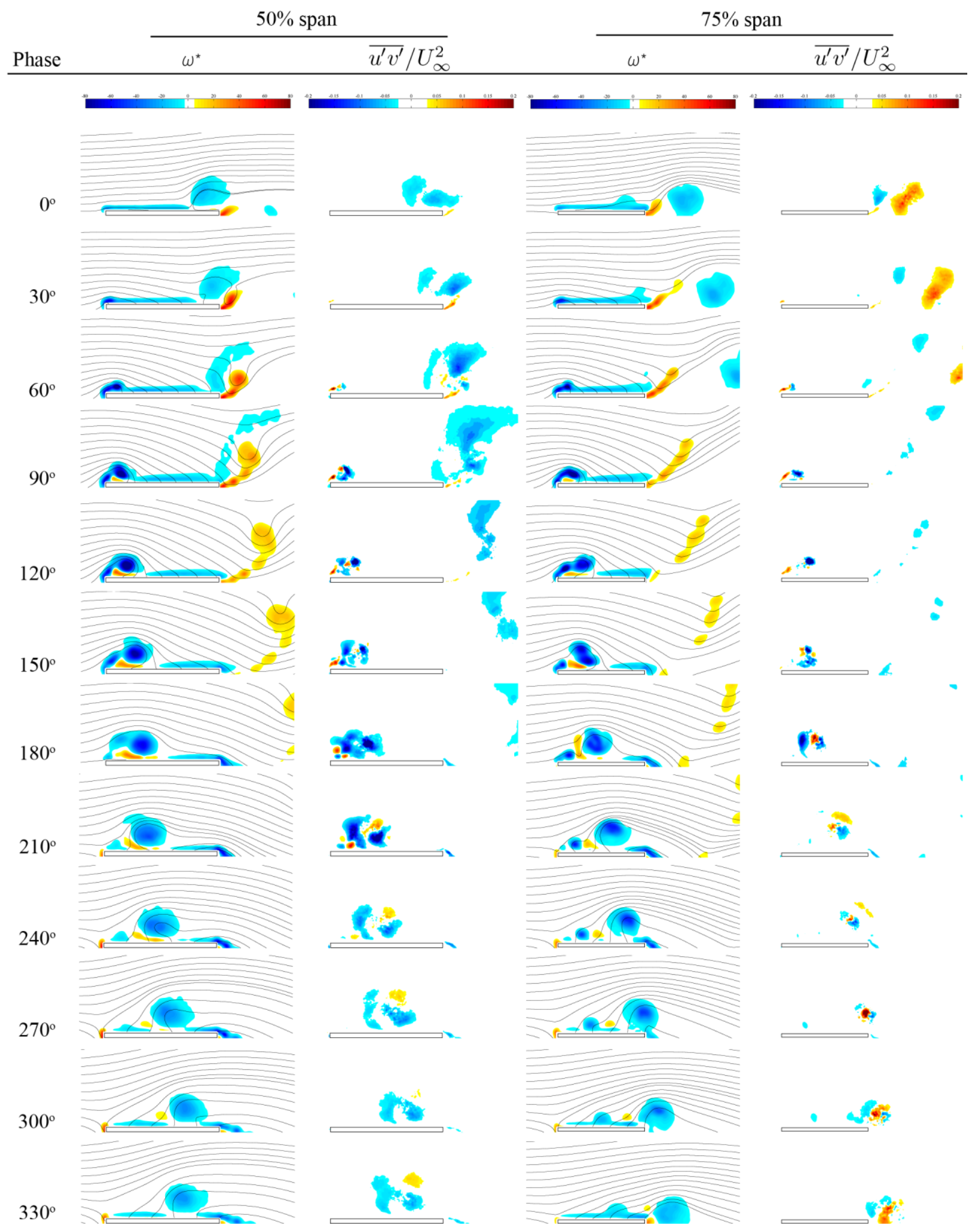

Figure 14. Normalized vorticity and Reynolds stress for the rigid aluminum model $\left(\Pi_{1}=90,240\right)$. PIV was performed at $50 \%$ and $75 \%$ span. Flow is from the left to right. 


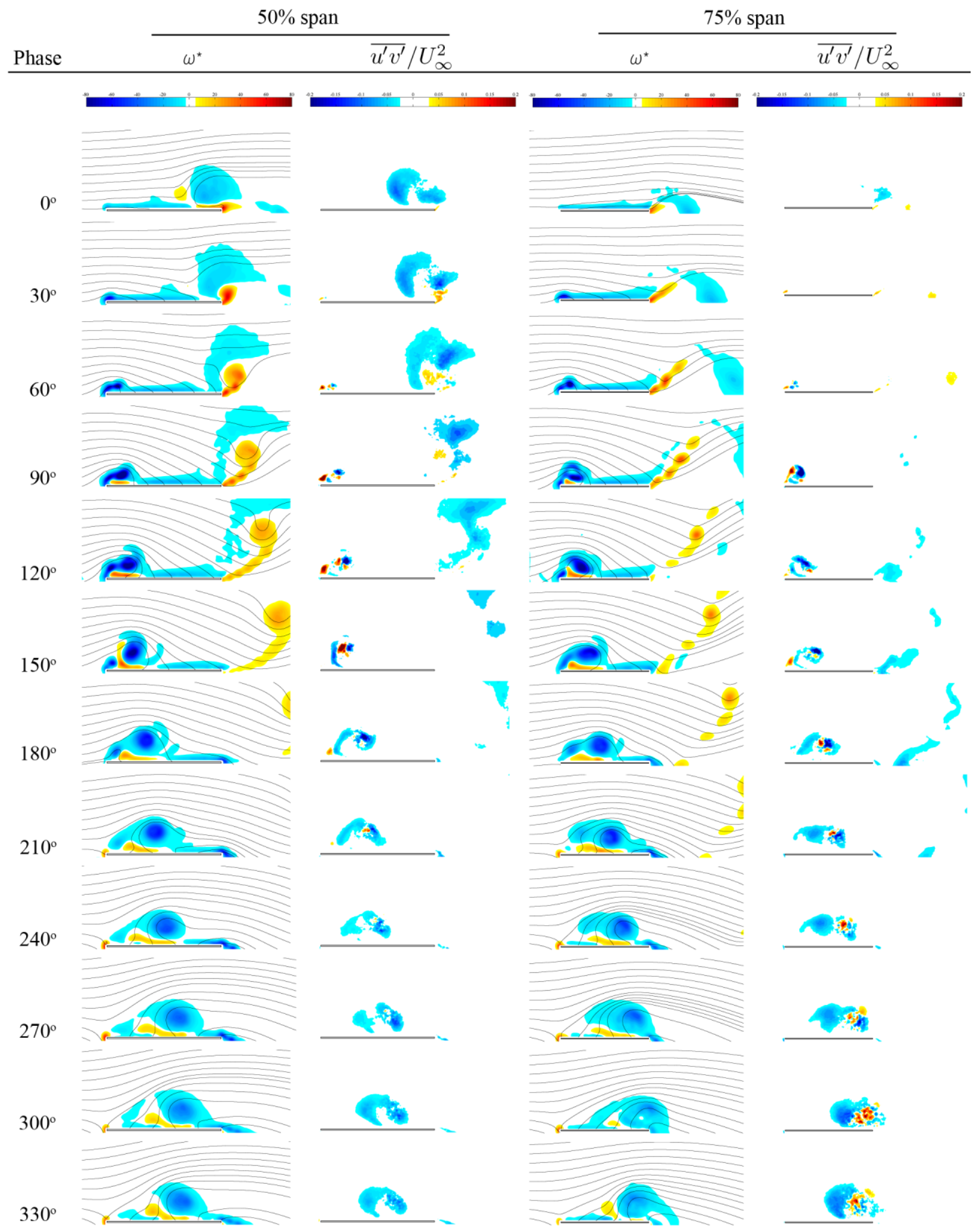

Figure 15. Normalized vorticity and Reynolds stress for the flexible aluminum model $\left(\Pi_{1}=1,460\right)$. PIV was performed at 50\% and $75 \%$ span. Flow is from the left to right. 


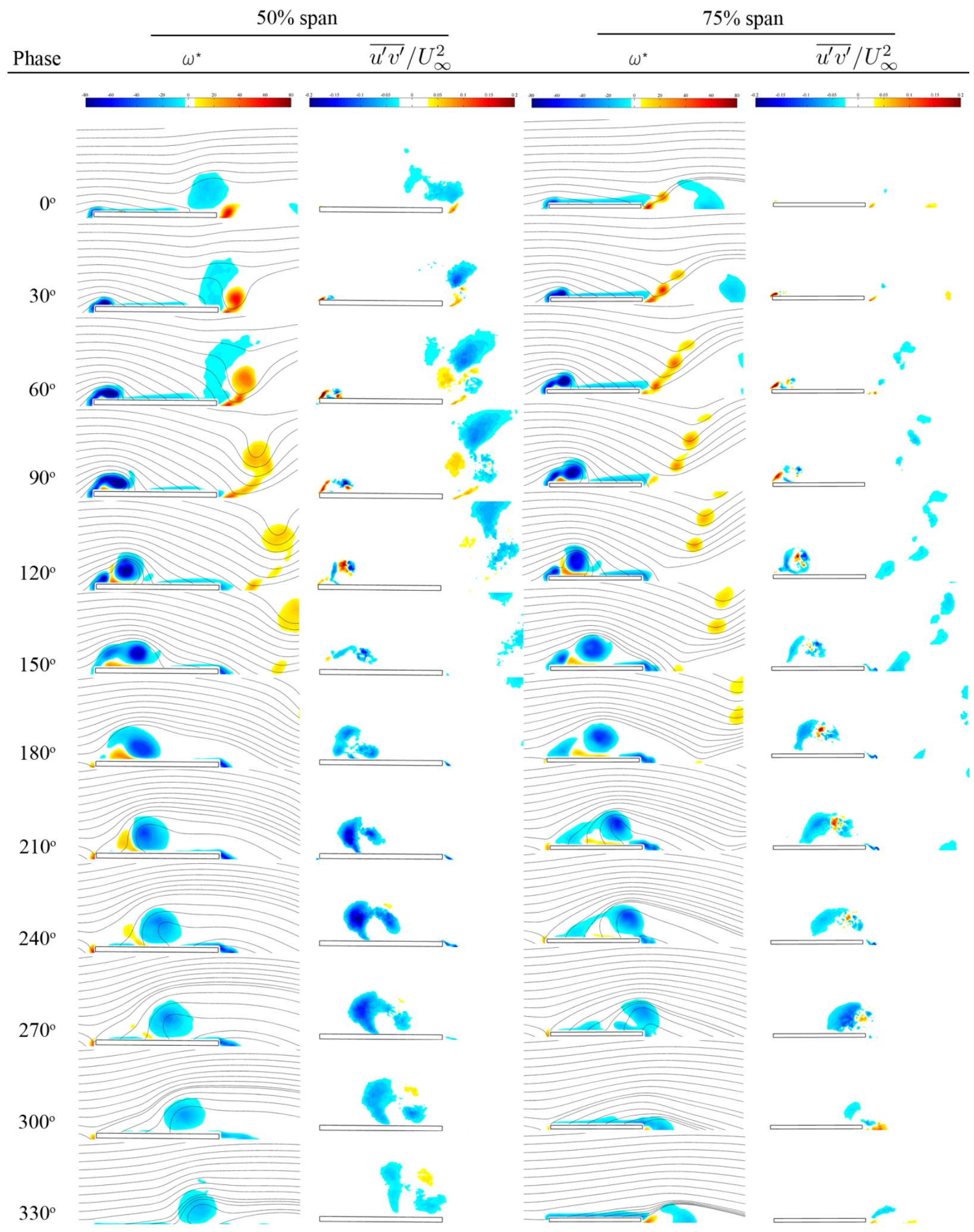

Figure 16. Normalized vorticity and Reynolds stress for the rigid HDPE model $\left(\Pi_{1}=1,960\right)$. PIV was performed at $50 \%$ and $75 \%$ span. Flow is from the left to right. 


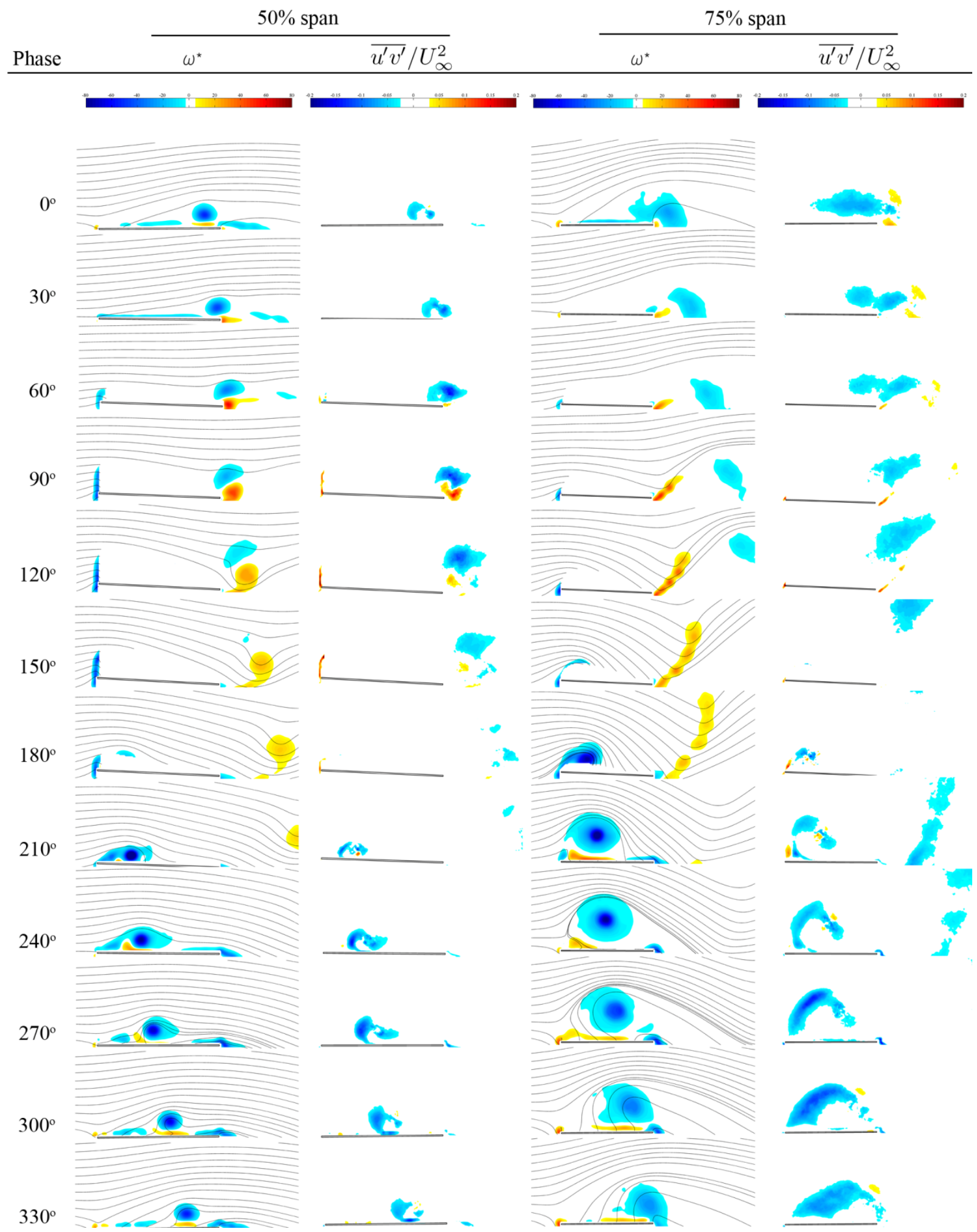

Figure 17. Normalized vorticity and Reynolds stress for the flexible HDPE model $\left(\Pi_{1}=30\right)$. PIV was performed at 50\% and 75\% span. Portions of the flowfields for phase 30 through 180 are masked because of structural deformations. Flow is from the left to right. 


\section{Flexibility Effect}

A flexibility effect was observed in all of the experimental measurements for the HDPE model with $\Pi_{1}=30$. The lower $\Pi_{1}$-value resulted in significant deformations. At spanwise locations farther away from the root, greater deformations and phase lags were measured with LDV. At $75 \%$ the structure deformed $40 \%$ the amplitude of motion with a $60^{\circ}$ phase lag between the structural deformation and the root-motion. The introduction of flexibility caused greater three-dimensional flow observed in the dye flow visualization. The three-dimensional flow was towards the tip which is in contrast to the rigid wing three-dimensional flow. A final observation of the flexibility effect is a larger leading edge vortex at the 75\% span location. The larger vortex at this location is caused by the greater plunge induced angle of attack.

\section{E. Thickness Effect}

When comparing the two aluminum models with the rigid HDPE model it was observed that the flowfield topologies correlated best when comparing similar model thicknesses and not the $\Pi_{1}$-parameter since no perceivable deflection was observed. This suggests that square edges yield thickness dependence for $1 \%-4 \%$ thickness-to-chord ratios.

This difference in flow topology is best observed as the leading edge vortex advects towards the trailing edge. Figure 18 compares the two alu minum and rigid HDPE models during the advection of the leading edge vortex at $50 \%$ span and phase $330^{\circ}$. In the comparison the difference in flow topology is highlighted by the thin aluminum model's greater positive vorticity generated by the advecting leading edge vortex. The aluminum and HDPE models of the same thickness have similar advection of the leading edge vortex that is in contrast with the thin alu minum model.

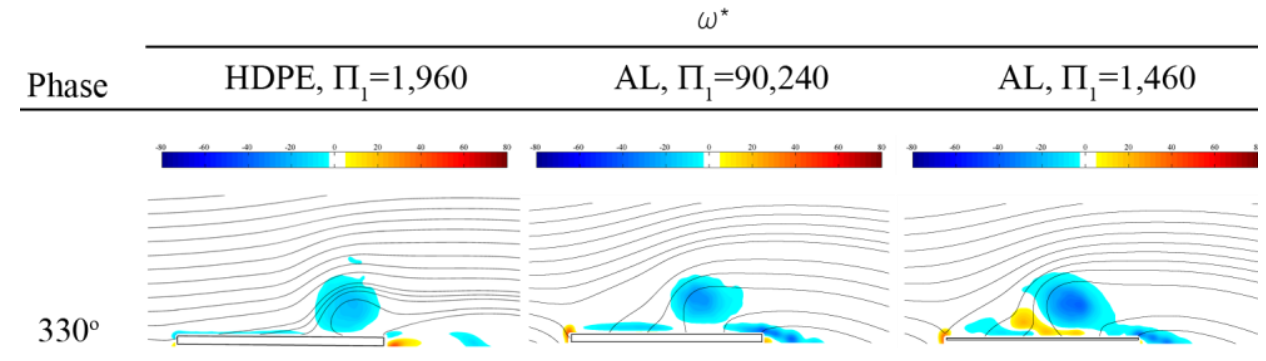

Figure 18. Vorticity comparison of the two aluminum (second and third columns) and rigid HDPE (first column) models at $50 \%$ span and phase $330^{\circ}$. The comparison evinces the thickness' effect on the flow topology of these nominally rigid models.

\section{Conclusions}

A study of spanwise-flexibility using $\Pi_{1}$-parameter has been performed. An elliptical flat plate with a 7.65 aspect ratio underwent a sinusoidal plunge in the University of Michigan water channel facility at Reynolds number 5,300 . The plunge motion is characterized by Strouhal nu mber 0.2 and dimensionless plunge amplitude 0.175.

Conclusions:

- For this model planform and aspect ratio the $\Pi_{1}$-parameter values greater than 1,460 yie ld no flexibility effect

- A noticeable flexibility effect was only observed for HDPE model with $\Pi_{1}=30$. The effect appeared in the dye flow visualization, LDV, and PIV.

$\circ$ This model's dye flow visualization evinced three-dimensional flow towards the wing tip which is opposite the direction of the nominally rigid models .

- The LDV measurement for this model yielded significant deformations highlighted by a $40 \%$ increase in displacement and a $29 \%$ increase in effective angle of attack at $75 \%$ span. This measurement also showed that the $25 \%-75 \%$ span lagged the root motion. The lag increased towards the tip where $50 \%$ span lags by $50^{\circ}$ phase and $75 \%$ span lags $70^{\circ}$ phase.

- The PIV measurement showed a larger leading edge vortex at $75 \%$ span than at $50 \%$ span. This increase in vortex size is caused by the increased plunge induced angle of attack.

19

American Institute of Aeronautics and Astronautics 
- A thickness effect was observed when comparing the PIV flow topologies of the two aluminum and rig id HDPE models. This effect indicates a flow dependence on the thickness-to-chord ratio over the $1 \%-4 \%$ range for square edges.

\section{Acknowledgements}

This work has been supported in part by the Air Force Office of Scientific Research's Multidisciplinary University Research Initiative.

\section{References}

${ }^{1}$ Shyy, W., Berg, M., \& Ljungqvist, D., "Flapping and Flexible Wings for Biological and Micro Air Vehicles," Progress in Aerospace Sciences, Vol. 35, 1999, 455-505.

${ }^{2}$ Koochesfahani, M.M., "Vortical Patterns in the Wake of an Oscillating Airfoil," AIAA Journal, Vol. 27, No. 9, 1989, 12001205.

${ }^{3}$ Lai, J.C.S., Platzer, M.F., "Jet Characteristics of a Plunging Airfoil,” AIAA Journal, Vol. 37, 1999, 1529-1537.

${ }^{4}$ Emblemsvag, J.E.., Suzuki,, R.., \& Candler, G.V., "Simulation of a Three-Dimensional Flapping Cantilever for Micro Air Vehicle Propulsion," AIAA 2003-4012.

${ }^{5}$ Anderson, J.M., Streitlien, K., Barrett, D.S., Triantafyllou, M.S. “Oscillating Foils of High Propulsive Efficiency,” Journal of Fluid Mechanics, Vol. 360, 1998, 41-72.

${ }^{6}$ Ol, M., Bernal, L., Kang, C., and Shyy, W., "Shallow and deep dynamic stall for flapping low Reynolds number airfoils," Experiments in Fluids, Vol. 46, Nr. 5, 2009, pp. 883-901

${ }^{7}$ Triantafyllou, M.S., "Hy drody namics of Fishlike Swimming," Annual Review of Fluid Mechanics, Vol. 32, 2000 , pp. 33-53.

${ }^{8}$ Wootton, R.J., "Support and Deformability in Insect Wings," Journal of Zoology London, Vol. 193, 1981, pp. 447-468.

${ }^{9}$ Shyy, W., Lian, Y., Tang, J., Viieru, \& Liu, H., 2008, Aerodynamics of Low Reynolds Number Flyers. Cambridge University Press.

${ }^{10}$ Shyy, W., Lian, Y., Tang, J., Liu, H., Trizila, P., Stanford, B., Bernal, L., Cesnik, C., Friedmann, P., \& Ifju, P., “Computational Aerody namics of Low Rey nolds Number Plunging, Pitching and Flexible Wings for MAV Applications," Acta Mech Sin, Vol. 24, 2008, 351-373.

${ }^{11}$ Heathcote, S., Gursul, I., "Flexible Flapping Airfoil Propulsion at Low Reynolds Numbers,” AIAA Journal, Vol. 45, No. 5, 2007, pp. 1066-1079.

${ }^{12}$ Heathcote, S., Wang, I., and Gursul, I., "Effect of Spanwise Flexibility on Flapping Wing Propulsion," Journal of Fluids and Structures, Vol. 24, No. 2, 2008, pp. 183-199.

${ }^{13}$ Aono, H., Chimakurthi, S. K., Wu, P., Sallstrom, E., Stanford, B.K., Cesnik, C. E. S., Ifju, P., Ukeiley, L., \& Shyy, W., “A Computational and Experimental Study of Flexible Flapping Wing Aerodynamics," AIAA 2010-554.

${ }^{14}$ Taylor, G. K., Nudds, R. L., and Thomas, A. L. R., "Fly ing and Swimming Animals Cruise at a Strouhal Number Tuned for High Power Efficiency," Nature, Vol. 425, No. 6959, pp. 707-711.

${ }^{15}$ Polytec Scanning Laser Vibrometer PSV 400 Hardware Manual, Poly tec GmbH, 2007.

${ }^{16}$ Salas, K. I., Cesnik, C. E. S., "Guided Wave Structural Health Monitoring Using CLo VER Transducer in Composite Plates," AIAA 2009-2106. 